\title{
Acute Invasive Fungal Rhinosinusitis: A Comprehensive Update of CT Findings and Design of an Effective Diagnostic Imaging Model
}

\author{
(D. E.H. Middlebrooks, C.J. Frost, R.O. De Jesus, T.C. Massini, I.M. Schmalfuss, and A.A. Mancuso
}

\begin{abstract}
BACKGROUND AND PURPOSE: Acute invasive fungal rhinosinusitis carries a high mortality rate. An easy-to-use and accurate predictive imaging model is currently lacking. We assessed the performance of various CT findings for the identification of acute invasive fungal rhinosinusitis and synthesized a simple and robust diagnostic model to serve as an easily applicable screening tool for at-risk patients.
\end{abstract}

MATERIALS AND METHODS: Two blinded neuroradiologists retrospectively graded 23 prespecified imaging abnormalities in the craniofacial region on craniofacial CT examinations from 42 patients with pathology-proven acute invasive fungal rhinosinusitis and 42 control patients proved negative for acute invasive fungal rhinosinusitis from the same high-risk population. A third blinded neuroradiologist decided discrepancies. Specificity, sensitivity, positive predictive value, and negative predictive value were determined for all individual variables. The 23 variables were evaluated for intercorrelations and univariate correlations and were interrogated by using stepwise linear regression.

RESULTS: Given the low predictive value of any individual variable, a 7-variable model (periantral fat, bone dehiscence, orbital invasion, septal ulceration, pterygopalatine fossa, nasolacrimal duct, and lacrimal sac) was synthesized on the basis of multivariate analysis. The presence of abnormality involving a single variable in the model has an $87 \%$ positive predictive value, $95 \%$ negative predictive value, $95 \%$ sensitivity, and $86 \%$ specificity $\left(R^{2}=0.661\right)$. A positive outcome in any 2 of the model variables predicted acute invasive fungal rhinosinusitis with $100 \%$ specificity and $100 \%$ positive predictive value.

CONCLUSIONS: Our 7-variable CT-based model provides an easily applicable and robust screening tool to triage patients at risk for acute invasive fungal rhinosinusitis into a disease-positive or -negative category with a high degree of confidence.

ABBREVIATIONS: AIFR = acute invasive fungal rhinosinusitis; NPV = negative predictive value; PPV = positive predictive value

$F^{\prime}$ ungal-related diseases of the nasal cavity and paranasal sinuses represent a broad spectrum of clinical entities, with acute invasive fungal rhinosinusitis (AIFR) being the most urgent and life-threatening. ${ }^{1-3}$ The primary risk factors for acquiring AIFR are neutropenia or dysfunctional neutrophils, and the most commonly reported predisposing conditions are hematologic malignancies, poorly controlled diabetes mellitus, chemotherapy, or organ transplantation. ${ }^{4-6}$ Although AIFR is a relatively rare disease, it carries a high mortality

Received August 26, 2014; accepted after revision January 15, 2015.

From the Department of Radiology (E.H.M., R.O.D.J., T.C.M., I.M.S., A.A.M.), University of Florida College of Medicine, Gainesville, Florida; Medical Imaging Consultants, Inc (C.J.F.), Gainesville, Florida; and North Florida/South Georgia Veterans Administration (T.C.M., I.M.S.), Gainesville, Florida.

Paper previously presented at: Annual Meeting of the American Society of Head and Neck Radiology, September 10-14, 2014; Seattle, Washington.

Please address correspondence to Erik H. Middlebrooks, MD, PO Box 100374 , Gainesville, FL 32610; e-mail: ehmiddlebrooks@gmail.com

- Indicates open access to non-subscribers at www.ajnr.org

三 Indicates article with supplemental on-line table

http://dx.doi.org/10.3174/ajnr.A4298 rate, with the largest published meta-analysis showing a mortality rate of approximately $50 \%{ }^{7}$ The high mortality rate underscores the importance of a timely diagnosis. Patients with AIFR limited to the nasal cavity have lower mortality rates, ${ }^{2}$ while intracranial extension is associated with twice the mortality. ${ }^{7}$ Accurate and easy-to-use predictive screening models that could help diagnose AIFR in a timely manner are currently lacking.

CT has long been considered an integral part of screening atrisk patients, despite the reported low specificity. ${ }^{8,9}$ The most commonly reported CT findings in early disease include severe unilateral nasal cavity mucosal thickening and soft-tissue infiltration of the maxillary periantral fat planes. ${ }^{8,10}$ Involvement of the pterygopalatine fossa has also been described. ${ }^{11}$ The most commonly affected areas are the middle turbinate, maxillary sinus, ethmoid air cells, and sphenoid sinus. ${ }^{2}$ The frontal sinus has been reported as the least frequently affected. ${ }^{2}$ Bone dehiscence, orbital invasion, and intracranial extension are more specific features of AIFR but are uncommon in early disease. ${ }^{1,8,9,12}$ These findings have also been implicated as indicative of advanced disease. ${ }^{10,11,13}$ 
A central purpose of this study was to characterize the imaging abnormalities of the nasal cavities, sinuses, and surrounding structures commonly associated with AIFR, as well as their incidence and predictive values. Previous institutional experience suggested that some features of AIFR are sparsely mentioned in or absent from the literature. This study was designed as a blinded retrospective study to comprehensively analyze the craniofacial region for changes on CT associated with AIFR. Because previous literature implicated the severity of the abnormality as a marker of AIFR, ${ }^{7}$ we applied ordinal scales to capture the degrees of nasal and paranasal mucosal disease and regional disease involvement. A key goal of our multivariate analysis was the synthesis of a simple and robust CT-based diagnostic model that could be deployed as a routine screening tool for at-risk patients. Ideally, this model would allow the diagnosis or exclusion of AIFR with a higher degree of confidence than any model previously suggested.

\section{MATERIALS AND METHODS}

\section{Patient Selection and Study Design}

Requirement for informed consent was waived in this Health Insurance Portability and Accountability Act-compliant retrospective study, which was approved by the university institutional review board. In our attempt to locate all patients undergoing work-up for possible AIFR, we searched the hospital archive for the term "invasive fungal" appearing in reports dating from January 1, 2007 to October 31, 2013, to identify potential study enrollees. Inclusion criteria were the following: 1) histopathologically proved invasive fungal rhinosinusitis meeting the European Organization for Research and Treatment of Cancer/Invasive Fungal Infections Cooperative Group and the National Institute of Allergy and Infectious Diseases Mycoses Study Group Consensus Group criteria for "proven" invasive fungal sinonasal disease, ${ }^{14}$ 2) a clinical time course of $<4$ weeks, and 3) CT imaging of the craniofacial region within the 5 days preceding biopsy or surgery. Forty-four patients met the inclusion criteria. Exclusion criteria were the following: 1 ) inadequate imaging by failure to cover the entirety of the craniofacial region (1 patient, $2 \%$ ), or 2 ) severe motion or beam-hardening artifacts. Forty-two such patients were enrolled as positive for AIFR. Our standard for patients positive for AIFR was histopathology confirming mucosal fungal invasion, which is the criterion standard. ${ }^{14}$

To differentiate findings related specifically to AIFR, we selected an appropriate control group of at-risk patients. These control patients came from the same at-risk population and had undergone endoscopy or surgery for high clinical suspicion of AIFR. Patients underwent endoscopy for elevated serum galactomannan (19\%, 8 of 42), suggestion based on imaging findings (33.3\%, 14 of 42), or clinical suspicion in the absence of serologic or imaging findings (ie, sinonasal symptoms clinically and persistent fever of unknown source [47.6\%, 20 of 42]). No patients were positive for $\beta$-D-glucan. Forty-two such patients were identified in reverse chronologic order with the inclusion criteria of having had an endoscopic visual survey of the nasal cavity with negative findings and/or negative histopathology results for invasive fungal disease, and CT imaging of the craniofacial region in the preceding 5 days. Exclusion criteria were the same as those for patients positive for AIFR. For the control group, all patients underwent, at minimum, a nasal endoscopic survey with negative findings. Biopsy or surgery was also performed in patients with suspicious lesions. All histopathology in the control group confirmed the absence of sinonasal fungal disease. Nasal endoscopy with biopsy of suspicious lesions has been validated as a screening tool $^{2}$ and served as the criterion standard for identification of control subjects in our study.

Clinical data were also collected from the medical record including surgical reports, histopathology reports, culture results, predisposing conditions, white blood cell count, absolute neutrophil count, and outcome. The absolute neutrophil count was tabulated only in patients with hematologic malignancy or bone marrow transplants. The cause of death was recorded as listed in the medical record.

\section{Imaging Protocol}

Eighty-one of the 84 studies were performed at the authors' hospital, using the institutional craniofacial CT protocol. According to our protocol, images were acquired as 1-mm-thick sections with spacing of $0.8 \mathrm{~mm}$ and an in-plane FOV from 170 to 190 $\mathrm{mm}$. Reconstructions using a bone algorithm were also performed at a section thickness of $0.75 \mathrm{~mm}$ with $0.5-\mathrm{mm}$ spacing. The scan was obtained in the axial plane from above the frontal sinuses through the hard palate. Multiplanar reformations were also completed in the coronal and sagittal plane. In 3 patients, only craniofacial studies performed at an outside institution were available for review. These studies were acquired with $2.5-\mathrm{mm}$ thickness with coronal and sagittal reformations. Both bone and soft-tissue algorithm images were obtained, and only 1 used intravenous contrast. Patients with clinical suspicion of intraorbital or intracranial involvement received intravenous iodinated contrast (75-mL iohexol, Omnipaque 350; GE Healthcare, Piscataway, New Jersey) at $0.8 \mathrm{~mL} / \mathrm{s}$ with imaging delayed 90 seconds after the injection of contrast. Contrast was administered in 11 of 42 patients with AIFR and 12 of 42 control patients.

\section{Image Analysis}

We used a blinded retrospective experimental design. Each study was completely anonymized before being loaded on an assigned PACS workstation. Two neuroradiologists independently interpreted the studies. Each reader had completed a 1-year Accreditation Council for Graduate Medical Education-approved neuroradiology fellowship and an additional dedicated 1-year fellowship in head and neck imaging. One reader (R.O.D.J.) had 3 years of experience interpreting head and neck imaging, and the second reader (T.C.M.) had 2 years of experience. A third reader (A.A.M.) with 35 years of experience in head and neck imaging was a tiebreaker for discrepancies by providing an ordinal value to replace the initial readers' results. The readers were blinded to all patient clinical information, histopathology results, and the number of AIFR cases versus controls. The studies were presented in randomized order to each reader.

The readers graded the amount of mucosal disease in each of the maxillary sinuses, frontal sinuses, sphenoid sinuses, anterior ethmoid air cells, posterior ethmoid air cells, anterior nasal cavity, posterior nasal cavity, and nasopharynx. Mucosal disease was 
Table 1: Demographics and clinical characteristics of both groups

\begin{tabular}{lccc}
\hline \multicolumn{1}{c}{ Characteristic } & AIFR Group & Control Group & $P$ Value \\
\hline Age $(y r)^{\mathrm{a}}$ & $49.5 \pm 21.2(2-85)$ & $46.7 \pm 21.2(2-75)$ & .54 \\
Male & 20 & 25 & \\
Female & 22 & 17 & \\
White blood cell count $\left(\times 1000 / \mathrm{mm}^{3}\right)^{\mathrm{a}, \mathrm{b}}$ & $0.9 \pm 1.5(0-6)$ & $4.5 \pm 12.4(0.1-65.6)$ & .3 \\
Absolute neutrophil count $\left(\times 1000 / \mathrm{mm}^{3}\right)^{\mathrm{a}, \mathrm{b}}$ & $0.51 \pm 1.2(0-4.9)$ & $1.8 \pm 4.0(0-20.3)$ & .66 \\
No. of patients $<500 / \mathrm{mm}^{3 \mathrm{~b}}$ & $60 \%(18 / 30)$ & $57.1 \%(20 / 35)$ & \\
No. of patients $<1000 \mathrm{~mm}^{3 \mathrm{~b}}$ & $83.3 \%(25 / 30)$ & $68.6 \%(24 / 35)$ & \\
Time from CT to treatment $($ days $)$ & $0.95 \pm 1.1$ & $0.83 \pm 0.82$ & .57 \\
Follow-up time $($ days) & $412.9 \pm 587.5(2-2836)$ & $346.7 \pm 402.4(18-2174)$ & .56 \\
Deceased from AIFR & $7^{\mathrm{c}}$ & 0 & \\
Deceased not from AIFR & 14 & 9 & \\
Not deceased & 20 & 33 & \\
Overall mortality & $52 \%(22 / 42)$ & $21 \%(9 / 42)$ & .003 \\
AlFR-related mortality & $17 \%(7 / 41)^{\mathrm{a}}$ & $0 \%(0 / 42)$ & \\
Non-AIFR related mortality & $34 \%(14 / 41)^{\mathrm{a}}$ & $21 \%(9 / 42)$ & \\
\hline
\end{tabular}

${ }^{\text {a }}$ Data are reported as mean, with range in parentheses.

${ }^{\mathrm{b}}$ Calculated only in patients with hematologic malignancy (including multiple myeloma) or bone marrow transplant. c One patient was excluded because the cause of death was uncertain, reported as died with concomitant fulminant liver failure and AIFR
Table 2: Prevalence of predisposing conditions for AIFR

\begin{tabular}{lcc}
\hline \multicolumn{1}{c}{ Predisposing Condition } & $\begin{array}{c}\text { Prevalence in } \\
\text { AIFR Group }\end{array}$ & $\begin{array}{c}\text { Prevalence in } \\
\text { Control Group }\end{array}$ \\
\hline Acute myelogenous leukemia & $42.9 \%(18 / 42)$ & $38.1 \%(16 / 42)$ \\
Diabetes & $28.6 \%(12 / 42)$ & $9.5 \%(4 / 42)$ \\
Other leukemia (non-AML) & $19.0 \%(8 / 42)$ & $21.4 \%(9 / 42)$ \\
Multiple myeloma & $7.1 \%(3 / 42)$ & $7.1 \%(3 / 42)$ \\
Solid organ malignancy & $7.1 \%(3 / 42)$ & $0 \%(0 / 42)$ \\
Solid organ transplant & $4.8 \%(2 / 42)$ & $9.5 \%(4 / 42)$ \\
Myelodysplastic syndrome & $2.4 \%(1 / 42)$ & $7.1 \%(3 / 42)$ \\
Non-Hodgkin lymphoma & $2.4 \%(1 / 42)$ & $11.9 \%(5 / 42)$ \\
None & $2.4 \%(1 / 42)$ & $0 \%(0 / 42)$ \\
\hline
\end{tabular}

Note:-AML indicates acute myelogenous leukemia.

Table 3: Cultured fungal pathogens in patients with AIFR

\begin{tabular}{lc}
\hline Fungal Species & No. of Cases \\
\hline Aspergillus sp & $42.9 \%(18 / 42)$ \\
Mucor sp & $23.8 \%(10 / 42)$ \\
Curvularia sp & $7.1 \%(3 / 42)$ \\
Fusarium sp & $2.4 \%(1 / 42)$ \\
Bipolaris sp & $2.4 \%(1 / 42)$ \\
Alternaria sp $^{\text {Unknown }}{ }^{\mathrm{a}}$ & $2.4 \%(1 / 42)$ \\
\hline
\end{tabular}

Note:-sp indicates species.

${ }^{a}$ Definitive speciation was not available in 8 cases.

graded on a scale of $0-5$ (eg, $0=$ normal, $1=<25 \%$ opacified, $2=25 \%-50 \%$ opacified, $3=50 \%-75 \%$ opacified, $4=75 \%-$ $100 \%$ opacified, $5=$ mucocele [ $100 \%$ opacified with expansion] ). The readers also recorded the presence of infiltration of the sphenopalatine foramen, pterygopalatine fossa, anterior periantral fat, posterior periantral fat, nasolacrimal duct, lacrimal sac, medial orbital fat, inferior orbital fat, and submucosa/bone of the hard palate on a $0-5$ scale on the basis of the subjective severity of involvement for the left and right sides separately. The presence of nasal septal mucosal ulceration was assigned as either "present" or "absent." The presence of subdural, epidural, or brain parenchymal extension, along with cavernous sinus involvement, abscess formation, bone dehiscence, arterial thrombosis, and/or venous using an expanded grading scale for extension beyond the sinus was to better elucidate minor discrepancies between readers. These values were analyzed as both graded variables and binary thrombosis, was similarly graded from 0 to 5 . The purpose of variables to ensure that no significant differences existed. The plane of the vertical process of the palatine bone defined the boundary between the sphenopalatine foramen and the pterygopalatine fossa.

There were 3 instances of disagreement (grading scale variation of $>1$ ) between the 2 primary readers that required reconciliation by the third reader. The specific disagreements included the presence of superior ophthalmic vein thrombosis $(0$ versus 2 ; final $=$ 0 ), involvement of pterygopalatine fossa ( 0 versus 3 , final $=3$ ), and cavernous sinus involvement ( 1 versus 5, final $=5$ ). All instances were in patients positive for AIFR.

\section{Statistical Analysis}

Basic data compilation and manipulation were performed in Excel (Microsoft, Bothell, Washington). Statistical analysis was performed by using JMP Software (SAS Institute, Cary, North Carolina). Specificity, sensitivity, positive predictive value, and negative predictive value were determined across ordinal ranges from binary (presence/absence) to the highest degree of opacity or disease involvement. Lateral data were used to assess lateral disease predominance. Otherwise, data from each measured region with laterality were consolidated to yield single ordinal estimates of disease prevalence or involvement. The resulting 23 variables were evaluated for intercorrelations and univariate correlations with AIFR and were further interrogated by using stepwise linear regression to elucidate the most salient predictors of AIFR. Variable entry into the regression required reducing Akaike Information Criterion, which is a measure of the relative quality of competing statistical models. ANOVA, $t$ tests, and nonparametric $\chi^{2}$ tests were used as appropriate to assess statistical differences between or among variables. The Cochran-Armitage Trend test was used as a modification to $\chi^{2}$ for ordinal variables to assess the relationships between the degree of opacity (or degree of involvement) and AIFR.

\section{RESULTS}

There were few statistical differences of note for any of the demographic or clinical variables collected (Table 1). In particular, there was no significant difference in the absolute neutrophil count of the 2 groups $(P=.66)$. The presence of blast crisis in 2 control patients increased the average absolute neutrophil count in the control group; however, this did not meet statistical significance. The control patients were all discharged and had a minimum of 18 days of follow-up (346.7 \pm 402.4 days) after negative endoscopy findings. No evidence of fungal sinusitis was present at the time of the last follow-up. The most prevalent predisposing conditions were leukemia, particularly acute myelogenous leukemia, and diabetes (Table 2). As expected from our sampling regimen, these predisposing conditions were represented in statistically similar manners between control patients and those with AIFR.

Aspergillus species (42.9\%) and Mucor species (23.8\%) were the most commonly isolated fungal pathogens (Table 3). Because 


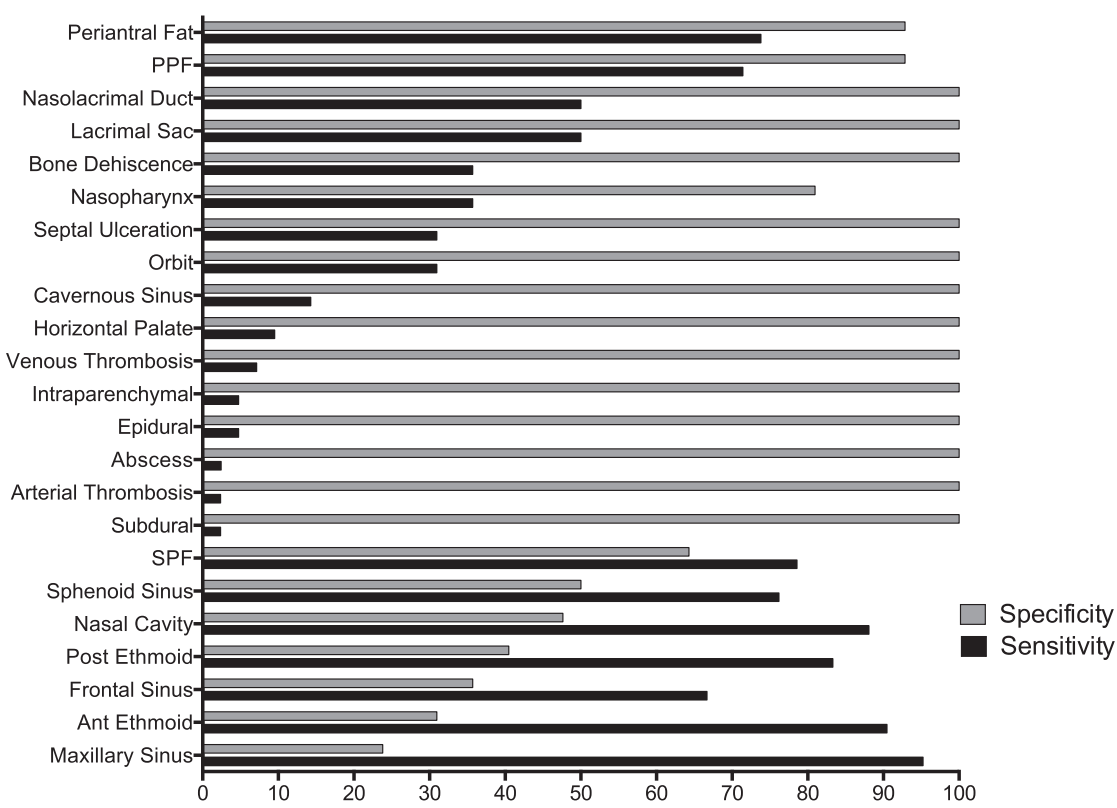

FIG 1. Sensitivity and specificity for all variables. PPF, pterygopalatine fossa; SPF, sphenopalatine foramen; Ant, anterior.
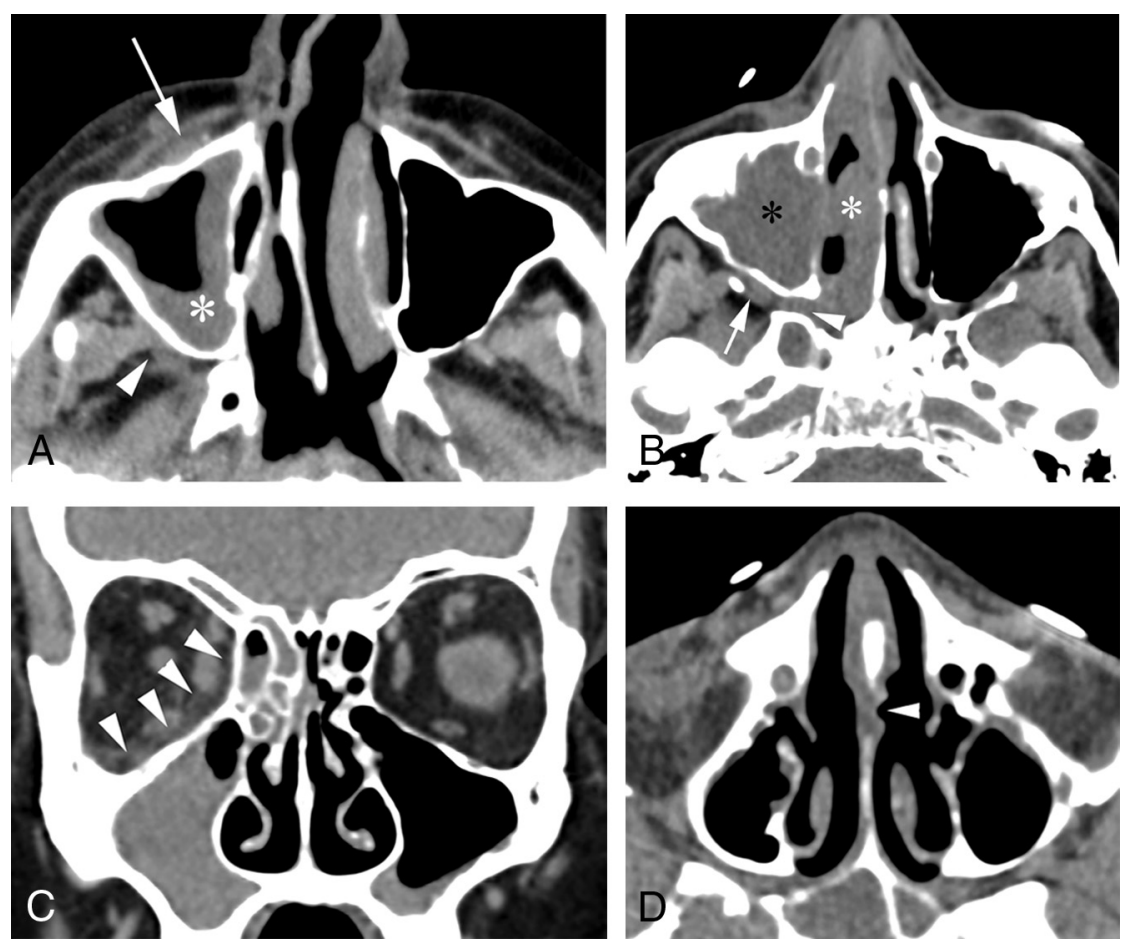

FIG 2. Examples of established findings in AIFR. A, Axial CT image shows unilateral mucosal thickening involving the right maxillary sinus (asterisk) with soft-tissue infiltration of the right anterior periantral fat (arrow) and the posterior periantral fat (arrowhead). B, Axial image in a different patient shows unilateral right nasal cavity (white asterisk) and maxillary sinus (black asterisk) mucosal thickening. Soft-tissue infiltration through the right sphenopalatine foramen and pterygopalatine fossa (arrowhead) is seen, as well as involvement of the right posterior periantral fat (arrow). C, Coronal CT in a third patient illustrates orbital involvement of AIFR with subtle infiltration of the right medial and inferior extraconal orbital fat (arrowheads), despite the absence of bone erosion. $D$, Axial CT shows a surgically proved subtle ulceration along the left side of the nasal septum (arrowhead) in a fourth patient.

fungal cultures commonly fail to have fungal growth, ${ }^{15}$ no definitive speciation could be determined in 8 patients (19\%). The overall mortality in the AIFR group was significantly higher than

1532 Middlebrooks Aug 2015 www.ajnr.org that in the control group (52\% versus $\left.21 \%, \chi^{2}=8.845, P=.003\right)$. The mortality related directly to AIFR was $17 \%$.

AIFR correlated most strongly with disease involvement in the pterygopalatine fossa $(r=0.64)$, periantral fat $(r=$ $0.61)$, nasolacrimal duct $(\mathrm{r}=0.52)$, and the lacrimal sac $(r=0.52)$. When considered as binary variables (ie, absence $[0] /$ presence $[1-5])$, these 4 variables displayed relatively high specificities (93\%-100\%) and sensitivities (50\%-74\%, Fig 1). A correlation of note is that sphenopalatine foramen involvement was present in $72 \%$ of patients positive for AIFR with pterygopalatine fossa involvement.

Thirteen variables had $100 \%$ specificity for AIFR, but only 5 of these had a sensitivity of $>30 \%$ (nasolacrimal duct, lacrimal sac, septal ulceration, orbital involvement, and bone dehiscence; Figs 2 and 3). The remaining variables with $100 \%$ specificity represented late-stage disease findings (epidural, subdural, abscess, venous thrombosis, arterial thrombosis, cavernous sinus involvement, intraparenchymal extension, and horizontal palate involvement; Fig 4). Variables related to the degree of opacity had relatively poor sensitivity and specificity as binary variables.

AIFR specificity increased significantly as a function of the degree of opacity in 6 of the 8 measured regions (Fig 5) - that is, the number of AIFRpositive cases was proportionally greater with higher severity of mucosal disease in the anterior nasal cavity $(Z=-3.99, P<.001)$, posterior nasal cavity $(Z=-4.51, P<.001)$, nasopharynx $(Z=-2.72, P=.003)$, sphenoid sinus $(Z=-2.89, P=.002)$, anterior ethmoid air cells $(Z=-3.53, P<.001)$, and posterior ethmoid air cells $(Z=$ $-3.91, P<.001$ ). In particular, 93\%$100 \%$ of the patients with $\geq 75 \%$ opacity (ordinal ranking, 3-5) in the nasal cavity or nasopharynx were positive for AIFR.

Laterality data were initially collected to determine whether a unilateral predominance existed. Unilateral AIFR was present in $78.6 \%$ of cases $\left(33 / 42, \chi^{2}=14.58, P<.001\right)$ with strong predilection of the disease for the right side. Only the right side was affected in $69.7 \%$ of unilateral cases $\left(23 / 33, \chi^{2}=5.26, P=.022\right)$. We did not find that laterality added additional predictive value to our models; 

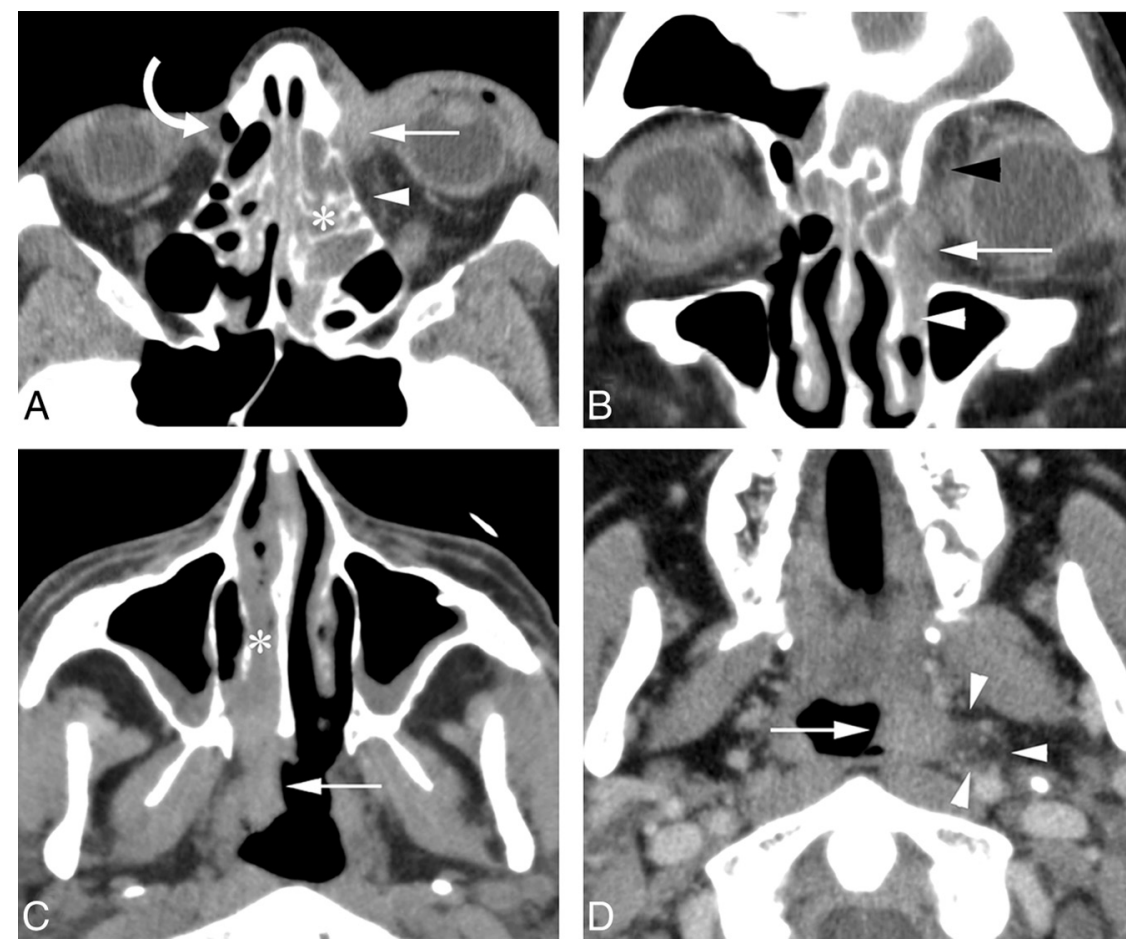

FIG 3. Illustration of less commonly described areas of AIFR, including the nasolacrimal duct, lacrimal sac, and nasopharynx. A, Contrastenhanced axial CT image shows soft-tissue thickening and inflammatory stranding in the area of the left lacrimal sac (white arrow) and in the medial orbit (white arrowhead). The normal right nasolacrimal duct (curved arrow) is identified for comparison. Asymmetric unilateral mucosal disease is also seen in the left ethmoid air cells (asterisk). B, Coronal image again shows thickening and inflammatory change in the left lacrimal $\mathrm{sac}$ (white arrow) and medial orbit (black arrowhead). Similar inflammatory changes are seen in the left nasolacrimal duct (white arrowhead). C, Axial CT in a different patient with AIFR with marked asymmetric mucosal thickening of the right nasal cavity (asterisk) and right nasopharynx (arrow).D, Axial contrast-enhanced CT in a third patient with marked asymmetric mucosal thickening in the left nasopharynx (arrow) and subtle inflammatory stranding involving the left parapharyngeal fat (arrowheads).
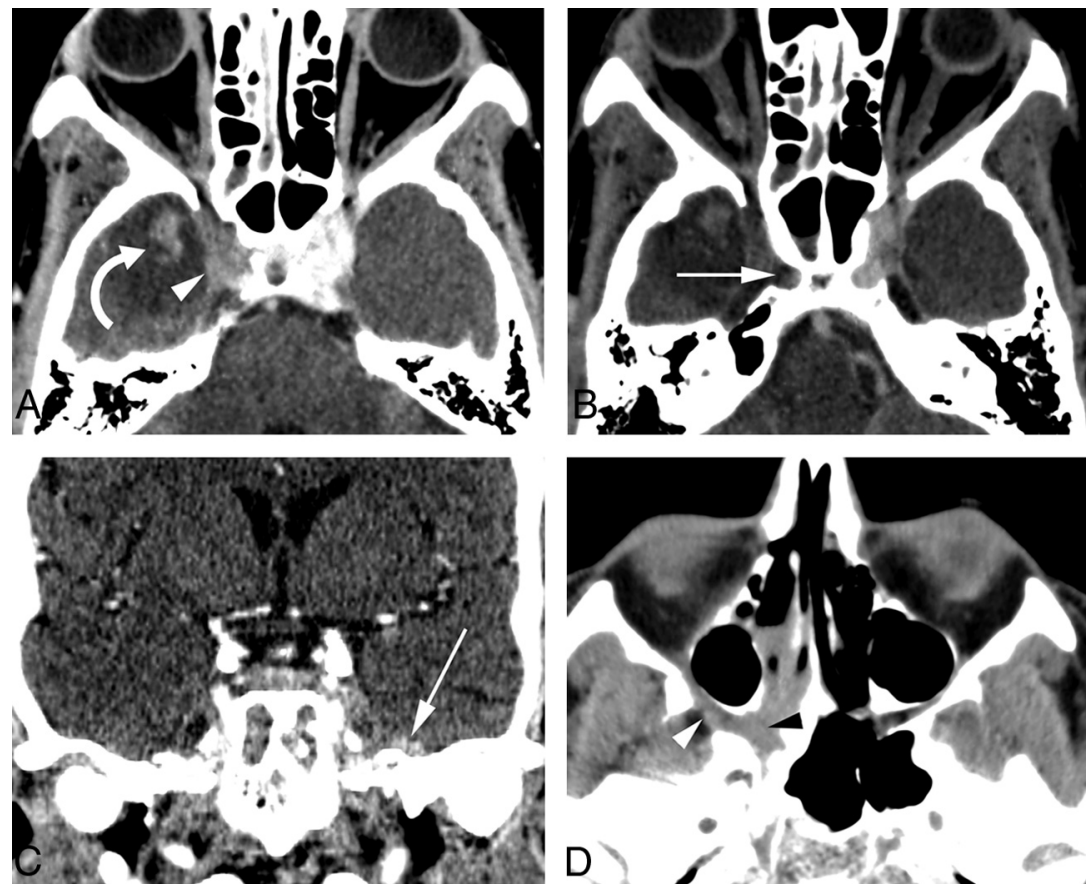

FIG 4. Examples of advanced manifestations of AIFR. $A$ and $B$, Axial postcontrast CT images show a filling defect in the right cavernous sinus (white arrowhead) with adjacent parenchymal hyperattenuation or focal area of enhancement (curved arrow) most consistent with an acute hemorrhagic infarction in the right anterior temporal pole or parenchymal involvement by AIFR, respectively. There is also partial thrombosis of the right internal carotid artery (white arrow). C, Postcontrast coronal CT in a different patient shows subtle left epidural thickening along the floor of the middle cranial fossa (white arrow). D, Axial noncontrast CT in a third patient shows soft-tissue infiltration of the right sphenopalatine foramen and pterygopalatine fossa (black arrowhead), with extension into the right orbital apex (white arrowhead). 

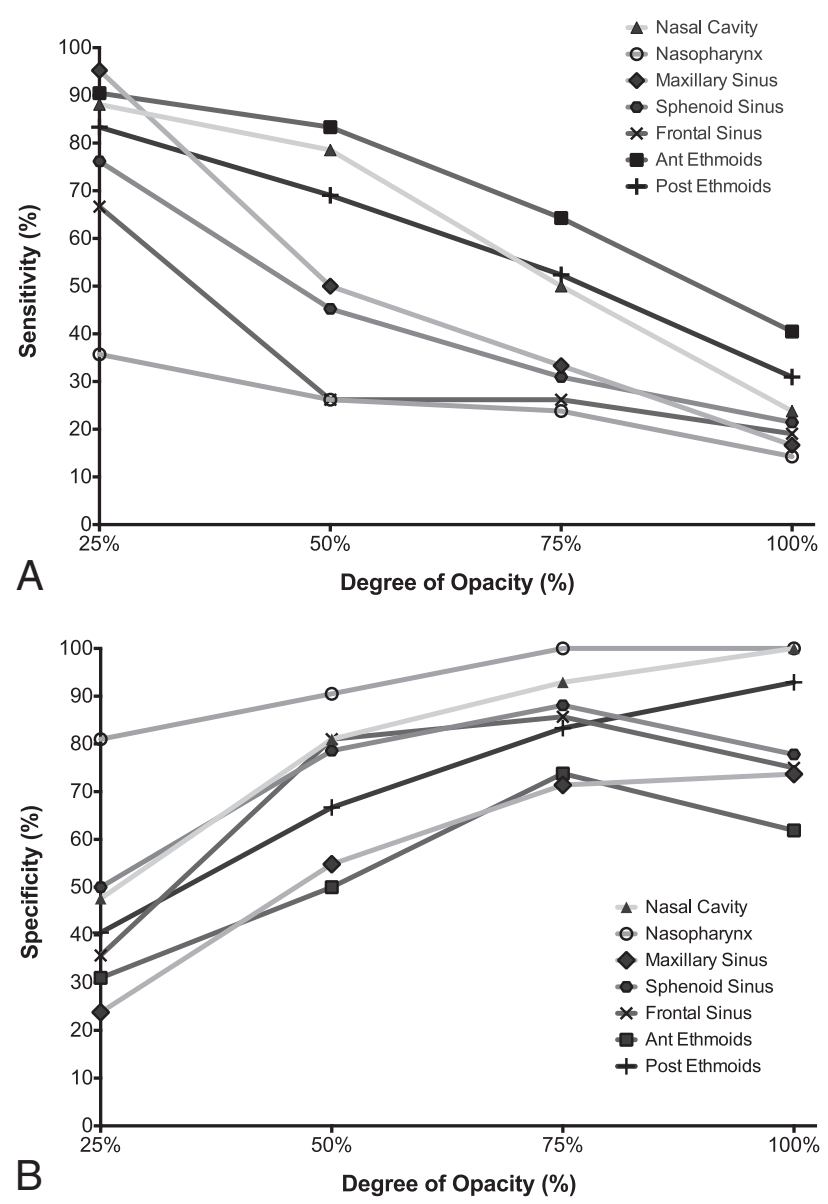

FIG 5. Correlation of the degree of opacity in the nasal cavity and paranasal regions with specificity $(A)$ and sensitivity $(B)$. Increasing opacity, particularly in the nasal cavity, nasopharynx, and posterior ethmoid air cells, has a strong correlation with specificity for AIFR. A decrease in sensitivity is also evident as the degree of opacity increases.

therefore, laterality data were consolidated for further analysis using the highest unilateral score.

We balanced statistical rigor of multivariate stepwise regression (minimizing Akaike Information Criterion) with the clinical reality of maximizing positive predictive value (PPV) and negative predictive value (NPV) to generate a 7-variable diagnostic model to predict AIFR (On-line Table). The top 4 variables in the model (periantral fat, pterygopalatine fossa, nasolacrimal duct, and lacrimal sac) were the most correlated with AIFR. These variables highly intercorrelated themselves and were present in most or all of the numerous statistical models generated during this evaluation. With respect to the 7-variable model, the presence of any single positive variable has an $87 \%$ PPV, 95\% NPV, 95\% sensitivity, and 86\% specificity. Moreover, a positive outcome in any 2 variables predicted AIFR with $100 \%$ specificity, $100 \% \mathrm{PPV}$, and $88.1 \%$ sensitivity. Our results show that $\geq 2$ of the 7 model variables were positive in $88 \%$ (37 of 42) of the patients with AIFR (Fig 6).

\section{DISCUSSION}

We present a simple-yet-accurate CT-based clinical model derived from a large single-institution study that can exclude or diagnose AIFR with a higher degree of confidence than suggested previously. ${ }^{8,9}$ A key attribute of this model is that within our clin-

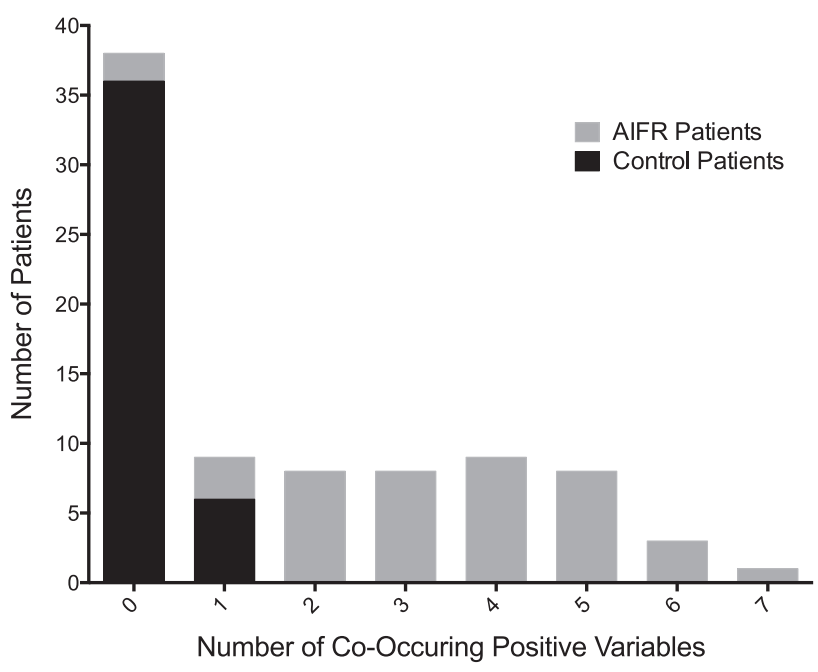

FIG 6. Illustration of the number of co-occurring positive variables (7-variable model) in patients with AIFR versus controls. The graph illustrates that most patients with AIFR have $>1$ positive area of involvement in the 7-variable model.

ical dataset, the involvement of any 2 of the 7 variables predicted AIFR with $100 \%$ specificity. The predictive power is also enhanced because $88 \%$ of patients with AIFR presented with findings captured by $\geq 2$ variables. This model has variables that have been previously ascribed to characteristic imaging findings for $\operatorname{AIFR}^{1,8,10,11}$ (periantral fat, bone dehiscence, orbital invasion, pterygopalatine fossa), as well as uncommonly described markers for AIFR such as nasolacrimal duct and lacrimal sac involvement. ${ }^{16,17}$

The power of the clinical model resides in the aggregate evaluation of all 7 variables because no individual variable had both high PPV and high NPV. For example, disease involvement in the periantral fat - an early indicator of AIFR ${ }^{10}$ — was the best individual predictor of AIFR in our study but, by itself, had a sensitivity of only $74 \%$. Bone dehiscence proved to be a specific marker for AIFR (100\% specificity) but has low sensitivity (35\%), which agrees with previously reported data. ${ }^{8}$ Because the fungi tend to spread through vascular channels or along nerves, ${ }^{18-21}$ extension outside of the sinus frequently occurs in the absence of bone destruction. Therefore, bone destruction alone is not a useful exclusionary criterion. This phenomenon likely explains the high correlation seen with involvement of the sphenopalatine foramen and ipsilateral pterygopalatine fossa, suggesting extension from the nasal cavity along either posterior superior nasal nerves or the sphenopalatine artery. This is in contradistinction to isolated involvement of the posterior periantral fat, which is more likely related to direct extension from the maxillary sinus along vascular channels.

Our study agrees with previous literature implicating severe nasal cavity mucosal thickening on CT as a common finding in patients with AIFR. ${ }^{8}$ This is noteworthy because of the relatively good prognosis when AIFR is limited to the nasal cavity. ${ }^{2}$ Unfortunately, unilateral nasal cavity disease has a low specificity though it is one of the more frequent findings in AIFR (78.6\% of patients in our study had unilateral predominant disease). Consequently, unilateral nasal cavity disease may not be a reliable individual predictor of AIFR. The correlation of AIFR with the 
severity of mucosal thickening was also present in areas outside the nasal cavity. We found a significant relationship between the incidence of AIFR and the degree of mucosal disease in 6 of 8 measured regions; however, incorporating such dependency on variable progression of mucosal disease proved difficult (and unnecessary). We opted for a clinical model that offered high PPV and high NPV, independent of opacity assignments for simpler clinical applicability.

To our knowledge, the mortality rate of our patient group (17\%) is one of the lowest published for a study of this scale, which suggests that these patients were diagnosed at a relatively favorable stage of disease. However, we were unable to identify any specific indictors of prognosis from our data - that is, in no region was disease involvement indicative of patient mortality. This was somewhat surprising because findings classically considered early-stage and late-stage factors were both present in our patients. If these monikers are accurate, patients presenting with so-called late-stage symptoms would be expected to have relatively high mortality, but this was not the case. For example, none of the 7 patients who died of AIFR presented with bone dehiscence. This outcome suggests caution in predicting a time course of disease or prognosis based on findings previously considered late-stage findings.

Our results show that CT is an effective screening tool for AIFR. Existing literature directly comparing MR imaging and CT found relatively higher sensitivity and PPV for AIFR by using MR imaging. This finding led to the recommendation that CT be considered a second-line technique. ${ }^{9}$ While our study populations were different, our CT-based predictive model nevertheless demonstrated higher sensitivity (95\% versus $86 \%$ ), specificity $(86 \%$ versus 75\%), and NPV (95\% versus 60\%) than these previously published MR imaging data. ${ }^{9}$ Our PPV estimates were similar (87\% versus 92\%). Moreover, our predictive model even produces higher sensitivity, specificity, and NPV than previously reported with CT. ${ }^{8,9}$ The NPV is of particular importance in screening studies because low NPV results in erroneously excluding a patient positive for AIFR. Thus, on the basis of our results, CT with application of our predictive model should be considered a primary technique for evaluating AIFR.

Several limitations in our study are noteworthy. First, the rarity of AIFR necessitated a retrospective study design to capture a large number of cases. Readers were blinded to all clinical data to minimize bias, but that is a concern in retrospective designs. Second, some selection bias is inherent because the control group all progressed to endoscopy or surgery, suggesting higher clinical suspicion of sinus disease. This bias would likely lead to underestimation of the NPV and overestimation of PPV. Third, we purposely selected a patient control group with predisposing conditions to AIFR so that our clinical model would be applicable to the interpreting radiologist evaluating possible AIFR among an atrisk patient population in a real-world clinical environment. Consequently, some caution is warranted in extrapolating our results to other patient groups in which AIFR is not the clinical concern.

\section{CONCLUSIONS}

We propose a CT-based model to help exclude or diagnose AIFR with a higher degree of confidence than suggested previously. ${ }^{9}$ Application of this proposed 7-variable model may improve eval- uation of potential AIFR in an at-risk population and serve as the basis for a subsequent prospective study.

\section{REFERENCES}

1. Aribandi M, McCoy VA, Bazan C 3rd. Imaging features of invasive and noninvasive fungal sinusitis: a review. Radiographics 2007;27: 1283-96

2. Gillespie MB, O’Malley BW Jr, Francis HW. An approach to fulminant invasive fungal rhinosinusitis in the immunocompromised host. Arch Otolaryngol Head Neck Surg 1998;124:520-26

3. Parikh SL, Venkatraman G, DelGaudio JM. Invasive fungal sinusitis: a 15-year review from a single institution. Am J Rhinol 2004;18: 75-81

4. Chakrabarti A, Denning DW, Ferguson BJ, et al. Fungal rhinosinusitis: a categorization and definitional schema addressing current controversies. Laryngoscope 2009;119:1809-18

5. Kasapoglu F, Coskun H, Ozmen OA, et al. Acute invasive fungal rhinosinusitis: evaluation of 26 patients treated with endonasal or open surgical procedures. Otolaryngol Head Neck Surg 2010;143: 614-20

6. Saghrouni F, Ben Youssef Y, Gheith S, et al. Twenty-nine cases of invasive aspergillosis in neutropenic patients. Med Mal Infect 2011;41:657-62

7. Turner JH, Soudry E, Nayak JV, et al. Survival outcomes in acute invasive fungal sinusitis: a systematic review and quantitative synthesis of published evidence. Laryngoscope 2013;123:1112-18

8. DelGaudio JM, Swain RE Jr, Kingdom TT, et al. Computed tomographic findings in patients with invasive fungal sinusitis. Arch Otolaryngol Head Neck Surg 2003;129:236-40

9. Groppo ER, El-Sayed IH, Aiken AH, et al. Computed tomography and magnetic resonance imaging characteristics of acute invasive fungal sinusitis. Arch Otolaryngol Head Neck Surg 2011;137:1005-10

10. Silverman CS, Mancuso AA. Periantral soft-tissue infiltration and its relevance to the early detection of invasive fungal sinusitis: CT and MR findings. AJNR Am J Neuroradiol 1998;19:321-25

11. Gamba JL, Woodruff WW, Djang WT, et al. Craniofacial mucormycosis: assessment with CT. Radiology 1986;160:207-12

12. Mossa-Basha M, Ilica AT, Maluf F, et al. The many faces of fungal disease of the paranasal sinuses: CT and MRI findings. Diagn Interv Radiol 2013;19:195-200

13. Centeno RS, Bentson JR, Mancuso AA. CT scanning in rhinocerebral mucormycosis and aspergillosis. Radiology 1981;140:383-89

14. De Pauw B, Walsh TJ, Donnelly JP, et al. Revised definitions of invasive fungal disease from the European Organization for Research and Treatment of Cancer/Invasive Fungal Infections Cooperative Group and the National Institute of Allergy and Infectious Diseases Mycoses Study Group (EORTC/MSG) Consensus Group. Clin Infect Dis 2008;46:1813-21

15. Chen CY, Sheng WH, Cheng A, et al. Invasive fungal sinusitis in patients with hematological malignancy: 15 years' experience in a single university hospital in Taiwan. BMC Infect Dis 2011;11:250

16. Halawa A, Yacoub G, Al Hassan M, et al. Dacryocystitis: an unusual form of Mucorales infection. J Ky Med Assoc 2008;106:520-24

17. Kapur R, Aakalu VK, August CZ, et al. Mucormycosis infection of the lacrimal sac. Ophthal Plast Reconstr Surg 2009;25:494-96

18. Margo CE, Linden C, Strickland-Marmol LB, et al. Rhinocerebral mucormycosis with perineural spread. Ophthal Plast Reconstr Surg 2007;23:326-27

19. McLean FM, Ginsberg LE, Stanton CA. Perineural spread of rhinocerebral mucormycosis. AJNR Am J Neuroradiol 1996;17:114-16

20. Orguc S, Yuceturk AV, Demir MA, et al. Rhinocerebral mucormycosis: perineural spread via the trigeminal nerve. J Clin $\mathrm{Neu}$ rosci 2005; 12:484-86

21. Frater JL, Hall GS, Procop GW. Histologic features of zygomycosis: emphasis on perineural invasion and fungal morphology. Arch Pathol Lab Med 2001;125:375-78 